\title{
A reduction in the implicit sense of agency during adolescence compared to childhood and adulthood
}

\author{
Ali Aytemur ${ }^{\mathrm{a}, *}$, Liat Levita ${ }^{\mathrm{b}}$ \\ a Department of Psychology, Manisa Celal Bayar University, Turkey \\ ${ }^{\mathrm{b}}$ Department of Psychology, University of Sheffield, UK
}

\section{A R T I CLE INFO}

\section{Keywords:}

Sense of Agency

Intentional Binding

Adolescence

Development

Libet Clock

Stream of Letters

\begin{abstract}
A B S T R A C T
Sense of agency (SoA), the fundamental feeling of control over our actions and their consequences, may show key developmental changes during adolescence. We examined SoA in childhood (9-10), mid-adolescence (13-14), late-adolescence (18-20) and adulthood (2528) using two tasks (Libet Clock and Stream of Letters). SoA was implicitly indexed by intentional binding that reflects the agency effect on action-outcome temporal association. We found age effects on the sub-processes in both tasks. In the Libet Clock task, where performance was more reliable, we observed a U-shaped developmental trajectory of intentional binding suggesting an adolescent-specific reduction in the experience of control. This study provides evidence for the developmental effects on the implicit agency experience and suggests adolescence as a critical period. We discuss the possible implications of these findings.
\end{abstract}

\section{Introduction}

Everyday voluntary actions are accompanied by an intrinsic feeling that we are in control of our actions and their outcomes. Sense of agency (SoA) refers to this fundamental experience, and successful construction of it by the brain is a key element of normal consciousness and mental health (Gallagher, 2000; Haggard, 2017; Pacherie, 2008). The experience of agency is central to everyday voluntary actions (Haggard, 2005, 2017) and impairments in this experience can underline some pathologies such as delusions of control in schizophrenia (Frith, Blakemore, \& Wolpert, 2000b; Synofzik, Thier, Leube, Schlotterbeck, \& Lindner, 2010; Voss et al., 2010; Voss, Chambon, Wenke, Kühn, \& Haggard, 2017). SoA is also important for societal and legal systems since it is closely tied to the individuals' responsibility for the outcomes of their actions (Frith, 2014; Haggard \& Tsakiris, 2009; Moretto, Walsh, \& Haggard, 2011). Adolescence might be an important period for SoA as brain regions associated with SoA such as the fronto-parietal network (Haggard, 2017; Sperduti, Delaveau, Fossati, \& Nadel, 2011) go through a significant maturational process during this developmental stage (Mills et al., 2016; Sowell, Thompson, Holmes, Jernigan, \& Toga, 1999b; Sowell, Thompson, Tessner, \& Toga, 2001; Zhou, Lebel, Treit, Evans, \& Beaulieu, 2015). Furthermore, adolescence is associated with a significant vulnerability in developing psychopathologies such as schizophrenia (Gomes, Rincon-Cortes, \& Grace, 2017; Harrop \& Trower, 2001) that involve distorted SoA (Frith et al., 2000b; Synofzik et al., 2010; Voss et al., 2017). However, a developmental approach for understanding possible changes in SoA and its functional implications have been largely neglected. To address this, the current study examined intentional binding, an implicit measure of SoA, in children (9-10), mid-adolescents (13-14) late-adolescents (18-20), and adults (25-28) using two different tasks, the Libet Clock and Stream of Letters.

Intentional binding is a temporal phenomenon where we observe a compression in the perceived interval between a voluntary action and its outcome, and these two events appear to occur closer in time than they actually are (Haggard, Clark, \& Kalogeras,

\footnotetext{
* Corresponding author at: Department of Psychology, Faculty of Science and Letters, Manisa Celal Bayar University, 45140 Yunusemre, Manisa, Turkey.

E-mail address: aali.aytemur@gmail.com (A. Aytemur)
} 
2002). It is thought that this temporal compression (intentional binding) represents the perceived linkage between actions and outcomes. Consequently, it has been considered to reflect the effect of agency on individual's perceived action-outcome temporal association and has been used as an implicit measure of SoA (Haggard, 2017; Moore \& Obhi, 2012). Notably, intentional binding consists of two sub-processes, action and outcome binding. Action binding is the subjective temporal shift of action towards outcome, and outcome binding is the subjective temporal shift of the outcome towards action, and the combination of these two elements constitutes the overall intentional binding effect (Haggard et al., 2002). That is, actions are reported to occur later, and outcomes are reported to occur earlier than they do which results in the overall temporal contraction between these two events. Action and outcome binding have been shown to be mediated by different mechanisms (Jo, Wittmann, Hinterberger, \& Schmidt, 2014; Moore \& Haggard, 2008; Moore, Ruge, Wenke, Rothwell, \& Haggard, 2010; Wolpe, Haggard, Siebner, \& Rowe, 2013). Outcome binding has been suggested to be mediated by predictive processes such as outcome prediction, whereas action binding has been suggested to be mediated by both predictive and retrospective (inference based) processes (Moore \& Haggard, 2008; Wolpe et al., 2013). Predictive processes are associated with internal motor processes such as action intention and outcome prediction (i.e., efferent copy) (Frith, Blakemore, \& Wolpert, 2000b; Haggard, 2017; Waszak, Cardoso-Leite, \& Hughes, 2012; Wolpert, Ghahramani, \& Jordan, 1995) whereas retrospective processes are associated with external sensory evidence processing and inferring agency retrospectively (Ebert \& Wegner, 2010; Moore \& Fletcher, 2012; Wegner and Wheatley, 1999).

In adults, intentional binding has conventionally been measured using the Libet Clock method (Haggard et al., 2002; Moore \& Obhi, 2012). This involves presenting a quickly rotating clock hand on an analogue clock face. Using this method, participants are asked to observe the clock and report the position of the clock hand at the time of an event of interest such as their own button press (action) or a beep sound (outcome). In a recent developmental study, an alternative method, the Stream of Letters, was used to compare intentional binding between children (mean age $=10, \mathrm{SD}=0.9$ ) and adults (mean age $=23$, SD $=1.4$ ), as the authors suggested the Libet Clock method may be difficult for children (Cavazzana, Begliomini, \& Bisiacchi, 2014). In this task, a random series of letters were presented sequentially on the computer screen, and participants needed to report the letter that was on the screen either when they made a button press (action) or when they heard a beep (outcome). Using this approach, Cavazzana et al (2014) found that children had reduced levels of intentional binding compared to adults suggesting a reduced implicit SoA in children. Cavazzana and collegues' (2014) finding raises a number of interesting questions such as when does implicit SoA become adult-like and how might it change throughout adolescence, a developmental period associated with significant structural and functional brain maturation processes (Ernst, 2014; Mills et al., 2016; Nagy, Westerberg, \& Klingberg, 2004; Sowell et al., 1999a; Zhou et al., 2015).

Adolescence could be an important developmental period for SoA because the brain regions which have been associated with different aspects of agency such as frontal areas that are involved in action selection (e.g., dorsolateral prefrontal cortex, DLPFC; Khalighinejad, Di Costa, \& Haggard, 2016; Wolpe et al., 2014) and action initiation (pre-supplementary motor area, pre-SMA and SMA; Haggard \& Whitford, 2004; Moore et al., 2010), and parietal regions that are involved in action and outcome monitoring (angular gyrus; Haggard, 2017; Khalighinejad \& Haggard, 2015; Voss et al., 2017) undergo significant structural and functional maturational changes during the transition from childhood to adulthood (Asato, Terwilliger, Woo, \& Luna, 2010; Casey, Getz, \& Galvan, 2008; Gogtay et al., 2004; Mills et al., 2016; Sowell et al., 2003; Zhou et al., 2015). Interestingly, there is a developmental lag in the maturation of parietal versus frontal cortical regions that are involved in different aspects of SoA processes, with parietal regions (action and outcome monitoring) showing earlier structural maturation compared to frontal regions such as the dorsolateral prefrontal cortex (action selection) (Gogtay et al., 2004; Mutlu et al., 2013; Sowell et al., 1999a; Sowell et al., 1999b). Prolonged age-dependent changes are also observed in sub-cortical regions, such as in the striatum from adolescence to adulthood (also see Asato et al., 2010), an area that is involved in voluntary movement initiation (Alexander \& Crutcher, 1990) and can consequently be involved in SoA. As these different brain regions mature at different rates age-dependent changes in SoA related processes might be evident.

To investigate this, the current study used intentional binding measured by two different tasks, Libet Clock and Stream of Letters, as a proxy of implicit SoA. This is because, although the Libet Clock and Stream of Letters seem to be somewhat similar, they do differ in at least three key ways. First, in the Stream of Letters task, random letters that are presented on the screen are unpredictable with regard to which letter would occur on the screen next (Cavazzana et al., 2014). In contrast, in the Libet Clock task, the motion of the clock hand is predictable with regard to where it could move in time (Libet, Gleason, Wright, \& Pearl, 1983). Quickly changing unpredictable letters might require greater attentional processes, which might make the method more difficult especially for children compared to a predictably moving clock hand. Furthermore, contextual predictability versus unpredictability could possibly influence the cognitive strategies used in the task (Moore \& Haggard, 2008; Synofzik, Vosgerau, \& Voss, 2013). Second, people might find meanings in the letters that are following each other (e.g., NP = no problem, MSC = Master of Science) which might increase processing demands whereas this would not be the case in Libet Clock. Third, in the Stream of Letters, each letter is presented on the screen for a certain duration (e.g., $150 \mathrm{~ms}$, Cavazzana et al., 2014) which would be needed for participants to recognize them; however, this might significantly reduce this method's temporal precision. In the Libet Clock, the motion of the clock hand is almost continuous ( $\sim 2 \mathrm{~ms}$ for each clock position, $2560 \mathrm{~ms}$ for one rotation, Haggard et al., 2002; Libet et al., 1983). Although the Libet Clock might provide temporal precision, it might be too fast and might reduce participants' accuracy when they are reporting the time at which certain events occurred. However, no study to date has examined intentional binding using both methods in the same study. By doing so, it would allow us to examine whether any age-dependent differences we observe in intentional binding are observed in both tasks, and also determine which of these tasks might act as a more reliable method to examine developmental changes in intentional binding. 
Consequently, the present study investigated the age-dependent changes in SoA from childhood through adolescence into adulthood in four age groups (9-10, 13-14, 18-20 and 25-28 years old). This study used intentional binding as a measure of SoA to benefit from its implicit nature (Haggard, 2017) and used both the Libet Clock and the Stream of Letters methods to measure intentional binding. Considering maturational changes in SoA related brain regions in the transition from childhood to adulthood we predicted changes in the developmental trajectory of implicit SoA, as measured by intentional binding. Cavazzana et al. (2014) suggested that intentional binding is reduced (a reduction in the temporal linkage between voluntary actions and their outcomes) in childhood and it increases in adulthood. However, what would happen during the transition from childhood to adulthood is unknown since, as discussed above, different brain regions associated with different aspects of agency experience mature at different rates. That is parietal regions (associated with action and outcome monitoring) mature earlier than frontal regions (associated with action selection and initiation) and how this developmental lag might be reflected in the developmental trajectory of implicit agency experience is not certain. As seen in the behavioural changes and brain maturation patterns during development (Casey, 2015), the developmental trajectory of intentional binding could be observed in different ways such as linear, adolescent-emergent or adolescent-specific. Hence, we would either see a linear increase in intentional binding with age (stronger temporal association between action and outcome), or it could increase up to a certain point in adolescence and then plateau (adolescent emergent), or it could show a U-shaped (or inverse U-shaped) developmental trajectory, with similar levels of intentional binding in childhood and adulthood and an adolescent-specific level of intentional binding.

\section{Methods}

\subsection{Participants}

An a priory G*power 3.1 analysis was run to determine appropriate sample size, which showed that a sample of 144 participants were needed to achieve 95\% power and a medium effect size. Overall, we were able to recruit 114 participants from four different age groups: children $(\mathrm{N}=21,11$ males, 9-10 years-old, mean age $=9.4, \mathrm{SD}=0.5)$, mid-adolescents $(\mathrm{N}=34,11$ males, $13-14$ years-old, mean age $=13.35, \mathrm{SD}=0.48)$, late-adolescents $(\mathrm{N}=26,11$ males, $18-20$ years-old, mean age $=18.9, \mathrm{SD}=0.77)$ and adults ( $\mathrm{N}=33,15$ males, 25-28 years old, mean age $=26.39, \mathrm{SD}=1.08)$. These age groups were chosen to allow us to track possible changes in SoA from childhood to adulthood. We chose the age range of our children group based on the work of Cavazzana et al, 2014, who tested 9-10 year old participants. We also wanted to examine possible changes in SoA in two different developmental time points during adolescence (middle and late). To that end, the age ranges for our two adolescent groups were based on previous work regarding the developmental trajectories of response inhibition (Steinberg et al, 2008), which shows important age related changes in the age ranges we choose for this study (13-14 \& 18-20). The age range for our adult group was based on the recent studies suggesting that adolescence ends in ones mid-twenties (Mills et al., 2014; Sawyer et al., 2018). Two participants were excluded based on a posteriorly defined exclusion criteria from further analysis due to the excessive intentional binding scores in the Libet Clock task that were more than two times of the delay duration $(2 \times 250 \mathrm{~ms})$ between the action and outcome and2 SD above from the group mean (one male participant from mid-adolescent and adult groups with respectively 551 and 589 ms intentional binding scores). Our initial strategy for participant recruitment was to recruit only unpaid volunteers. However, since the number of participants recruited without monetary compensation was small ( $\mathrm{n}=22$ in mid-adolescents and $\mathrm{n}=13$ in adults), we decided to either offer a monetary reward $(£ 5)$ for participation $(n=58)$, or course credit $(n=21)$. All participants (and their parents if they were under 18) gave consent before the experiment. The study was approved by the University of Sheffield, Department of Psychology Ethics Committee.

\subsection{Materials and Experimental procedure}

For the experimental task, participants sat in front of a computer screen (144 Hz, 24-inch, iiyama ProLite GB2488HSU-B1) in a dimly lit room. PschToolbox 3 (Kleiner et al., 2007) was used to present the two intentional binding tasks. Since timing was critical for this study, participants made button presses using a custom-built button box connected to an Arduino micro-controller ( $<2$ ms latency; Schubert, D'Ausilio, \& Canto, 2013). The auditory stimulus (100 ms duration, $1000 \mathrm{~Hz}, \sim 85 \mathrm{~dB}$ ) was presented using a Sennheiser HD 202 Stereo over-ear headphone.

The same procedures introduced by Haggard et al. (2002) for the Libet Clock and Cavazzana et al. (2014) for the Stream of Letters tasks were followed. The order of the tasks was counterbalanced across participants and the order of the conditions (Baseline Action, Baseline Beep, Experimental Action and Experimental Beep, see below) within each task was randomised. At the end of each task, we asked the youngest (children) and the oldest (adults) age groups to rate the difficulty of the task they just completed on a scale from 1 (very easy) to 7 (very difficult), due to a procedural error this data was not collected from adolescent participants.

\subsubsection{Libet Clock task}

The Libet Clock task followed the same procedure as described by Haggard et al. (2002). In this task, participants had to observe a clock hand (12 mm) rotating on an analogue clock (Fig. 1B) in four different conditions Baseline Action, Baseline Beep, Experimental Action and Experimental Beep (each condition consisted of 33 trials). To start each trial participants had to press the space button with their left hand when they were ready. After the start of each trial, the clock hand appeared at a random position on the clock face, and started rotating (duration of one rotation was $2520 \mathrm{~ms}$ ). During the Baseline Action condition participants were instructed 
A)

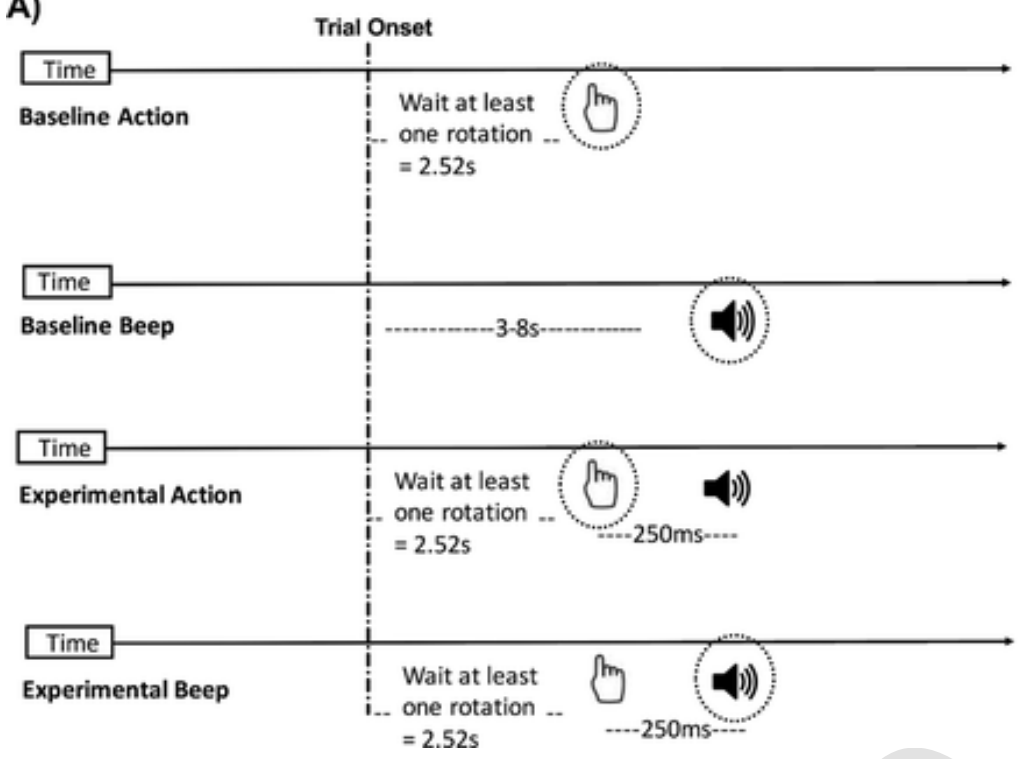

B)

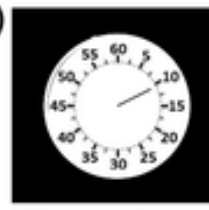

What was the position of the clock hand at the time when ....?

C)

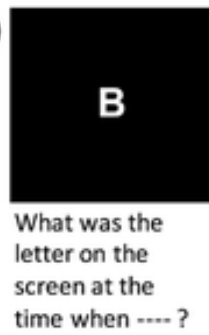

Fig. 1. (A) The four conditions in both Libet Clock and Stream of Letters tasks. In the baseline conditions participants reported the time of either their button press (Baseline Action) or a beep sound (Baseline Beep) that occurred at a random time between 3 and $8 \mathrm{~s}$ after trial onset. In the experimental conditions participants again had to report either the time of their button press (Experimental Action) or beep sound (Experimental Beep). However, this time, actions were followed by the beep sounds with $250 \mathrm{~ms}$ delay. Participants reported the time of the events in dashed circles in these four conditions. Participants started each trial at a time of their own choosing by pressing space button on the keyboard with their left hand. (B) The Libet Clock that was used to report the time of the events. Participants were required to report the position of the clock hand at the time of an event of interest. (C) An example of one of the changing letters in the Stream of Letters task. Participants were required to report the letter on the screen at the time of an event of interest. Size of the clock and letter are not to scale.

to make a button press with their right index finger at a time of their own choosing (Fig. 1A) and they were then asked to judge the clock position when they made the button press. They were also instructed not to respond in a stereotyped fashion, at a pre-decided clock position or during the first rotation of the clock hand. During the Baseline Beep condition, participants were asked to judge the position of the clock at the time of a beep sound that was triggered by the computer at a random time between 3 and $8 \mathrm{~s}$ after the trial onset. In the two experimental conditions, participants made a button press and after 250 ms delay, they heard a beep sound. In these conditions, unlike baseline conditions, button presses and beep sounds were coupled (action-outcome). In the Experimental Action condition participants were asked to judge the position of the clock at the time when they pressed the button and in the Experimental Beep condition, participants were asked to judge the position of the clock at the time of the beep sound. In all conditions, the clock hand stopped at random intervals (1.5-2.5 s) after the event of interest occurred (button press or beep sound). Participants were asked to report the position of the clock hand at the end of each trial using the keyboard. The clock face (without the clock hand) was on the screen until they made their response.

\subsubsection{Stream of Letters task}

Stream of Letters task followed the same procedure described by Cavazzana et al. (2014). This task involved presenting sequential series of randomly changing letters on the screen (white, capital consonants, 100-point font size, Fig. 1C). Random numbers (from 0 to 9) were presented before the letters for $\sim 2.5 \mathrm{~s}$ (corresponding to one rotation of the Libet Clock). Each number and letter were presented for $150 \mathrm{~ms}$ with no inter-stimulus intervals. Participants were instructed to observe the letters and asked to report the letter on the screen at the time of a button press or beep. The procedure and conditions were same as Libet Clock task. In the conditions when participants were making button presses, they were asked to wait before making a button press until they started to see the letters appearing on the screen randomly after the numbers (same as waiting for one rotation in the Libet Clock task). The stream of letters stopped at a random interval (1.5 - $2.5 \mathrm{~s})$ after the event of interest occurred. Participants were presented with a response map (corresponds to the clock face presented at the end in Libet Clock method) which included the letter at the time of the event, two letters before and two letters after it. The order of these letters was randomised when they were presented on the screen (Cavazzana et al., 2014).

\section{Data analysis}

\subsection{Judgement error}

The mean scores of judgment errors (JE), which is the difference between the reported and actual time of the specified events (button press or beep), was calculated for each condition. That is, JEs were calculated as the difference between reported clock position and actual clock position multiplied by 42 (42 ms was the duration of one clock position) for Libet Clock task and the differ- 
ence between reported letter and actual letter in a sequence of letters multiplied by 150 (each letter stays on the screen for 150 ms) for Stream of Letters task. The first three trials of each condition were considered as practice and were excluded from the data analysis (Cavazzana et al., 2014). For all groups, judgement errors that were 2SD above and below the average of each condition (Engbert, Wohlschläger, Thomas, \& Haggard, 2007; Wolpe et al., 2013) were excluded from further analysis for both tasks. Trials excluded in percentage: Libet Clock task, Children: 5.1\%, Mid-Adolescents: 4.4\%, Late-Adolescents: 5\% and Adults: 3.8\%; Stream of letters task, Children: 2.5\%, Mid-Adolescents: 4.5\%, Late-Adolescents: $4.1 \%$ and Adults: $4.8 \%$.

\subsection{Intentional binding}

Intentional binding score was calculated as commonly reported in the literature (Haggard et al., 2002). Action binding was calculated by subtracting JE in Baseline Action from Experimental Action condition (Fig. 2). Outcome binding was calculated by subtracting the Baseline Beep from Experimental Beep condition. Action and outcome binding represent the perceived temporal shift of action or outcome towards one another respectively in comparison to a baseline. Then, overall intentional binding was calculated as the combination of action and outcome binding which represents the effect of agency on the perceived action-outcome temporal relationship (Fig. 2). Judgement error and intentional binding scores of conditions across age groups and tasks can be seen in Table 1.

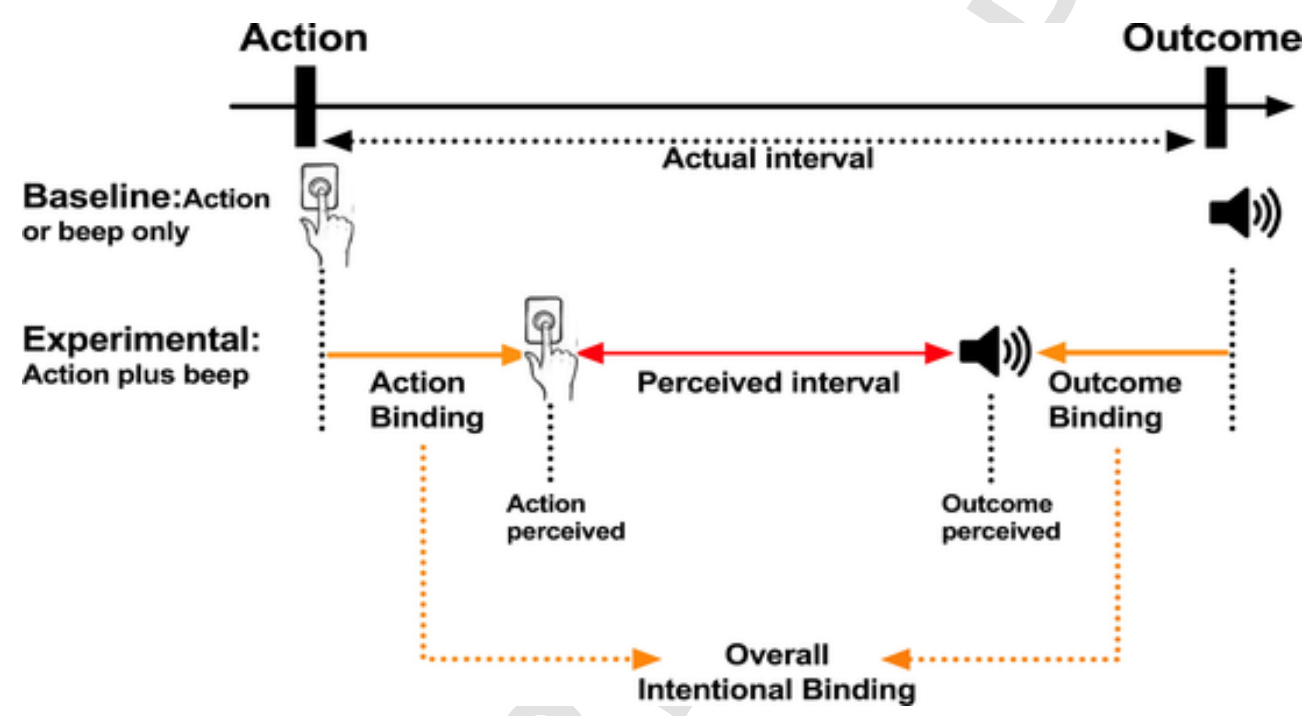

Fig. 2. Schematic illustration of intentional binding effect. In the baseline conditions, there is either action or beep sound (outcome) and participants report the time of these events. In the experimental conditions, actions are coupled with beep sounds (action + outcome) and participants report the time of these events. Action binding represents the perceived temporal shift of action towards outcome. Similarly, outcome binding represents the perceived temporal shift of outcome towards action. Overall intentional binding is calculated by combining action and outcome binding and represents the perceived compression of the interval between these two events.

Table 1

Judgement errors and intentional binding scores in millisecond for the Libet Clock (LC) and Stream of Letters (SoL) tasks across age groups and conditions. Standard deviations (SD) presented in brackets.

\begin{tabular}{|c|c|c|c|c|c|c|c|c|}
\hline & & Baseline Action & Exp. Action & Baseline Beep & Exp. Beep & Action Binding & Outcome Binding & Overall Binding \\
\hline \multirow[t]{2}{*}{ Pre-Adolescent } & LC & $27(98)$ & $88(76)$ & $20(67)$ & $-130\left(\begin{array}{lll}1 & 1 & 7\end{array}\right)$ & $61(92)$ & $-150\left(\begin{array}{lll}1 & 0 & 7\end{array}\right)$ & $212\left(\begin{array}{lll}1 & 3 & 0\end{array}\right)$ \\
\hline & SoL & $16(66)$ & $41(90)$ & $62(55)$ & $-79(86)$ & $25(52)$ & $-142(72)$ & $167(70)$ \\
\hline \multirow[t]{2}{*}{ Mid-Adolescent } & LC & $49(85)$ & 119(1 112$)$ & $2(43)$ & $-92(83)$ & $69(85)$ & $-94(64)$ & $163(94)$ \\
\hline & SoL & $46(76)$ & $99\left(\begin{array}{lll}1 & 0 & 5\end{array}\right)$ & $10(35)$ & $-57(70)$ & $53\left(\begin{array}{lll}1 & 0 & 1\end{array}\right)$ & $-67(68)$ & $120\left(\begin{array}{lll}1 & 2 & 2\end{array}\right)$ \\
\hline \multirow[t]{2}{*}{ Late-Adolescent } & LC & $47(56)$ & $94(69)$ & $-13(63)$ & $-67(94)$ & $47(62)$ & $-54(80)$ & 101 (89) \\
\hline & SoL & $19(67)$ & $89\left(\begin{array}{lll}1 & 0 & 4\end{array}\right)$ & $10(43)$ & $-88(75)$ & 70 (94) & $-99(78)$ & $169(126)$ \\
\hline \multirow[t]{2}{*}{ Adult } & LC & $50(51)$ & $111(78)$ & $15(54)$ & $-109(93)$ & $61(47)$ & $-125(86)$ & $186(92)$ \\
\hline & SoL & $34(67)$ & $93(95)$ & $-4(43)$ & $-54(83)$ & $59(70)$ & $-50(82)$ & $110\left(\begin{array}{lll}1 & 1 & 3\end{array}\right)$ \\
\hline
\end{tabular}




\section{Results}

\subsection{Intentional binding}

\subsubsection{Developmental differences in overall intentional binding}

To investigate the developmental trajectory of implicit SoA, a mixed model ANOVA was conducted on the intentional binding scores with Task as a within and Age-Group as a between subject factor. There was a trend level main effect of Task $\left[F(1,108)=3.48, p=0.06, \eta p^{2}=0.031\right]$ and a significant interaction of Task with Age-Group $[F(3,108)=6.09, p=0.001$, $\eta p^{2}=0.145$. Our post-hoc analysis showed that significant developmental differences were found when intentional binding was measured with Libet Clock $\left[F(3,108)=5.52, p=0.01, \eta p^{2}=0.133\right]$. As can be seen in Fig. 3A, when measured with Libet Clock, intentional binding scores were high in children, and steadily decreased with age during adolescence, after which intentional binding increased again to the children's level in adulthood. Pairwise comparisons showed that late-adolescents had significantly smaller intentional binding scores compared to children $[t(45)=-3.45, p=0.001]$, mid-adolescents $[t(57)=-2.57, p=0.013]$ and adults $[t(56)=-3.54, p<0.001]$, and mid-adolescents did not have significantly different intentional binding than children $[t(52)=-$ $1.58, p=0.11]$. Adults' intentional binding scores did not significantly differ from children $[t(51)=-0.83, p=0.41]$ or mid-adolescents $[t(63)=0.99, p=0.32]$. In contrast, no developmental differences were observed when intentional binding was measured with Stream of Letters task $\left[F(3,108)=2.05, p=0.110, \eta p^{2}=0.054\right]$ (Fig. 3B).

Further following the significant interaction between Task and Age-Group, pairwise comparisons showed that, in children, intentional binding measured with either task did not significantly differ $[t(20)=1.34, p=0.19]$. However, in adults, intentional binding measured with Libet Clock was significantly greater than that measured with the Stream of Letters task $[t(31)=3.35, p=0.002]$, and this pattern was similar in mid-adolescents, but was only marginally significant $[t(32)=1.76, p=0.08]$. Notably, a different pattern was observed in late-adolescents where intentional binding measured with Stream of Letters was significantly higher than intentional binding measured with Libet Clock $[t(25)=-3.04, p=0.005]$.

\subsubsection{Developmental differences in action and outcome binding}

We further investigated possible age-dependent differences in action and outcome binding separately, as it has been suggested that they are associated with different processes and hence might show different developmental trajectories (Moore \& Haggard, 2008; Wolpe et al., 2013; Moore et al., 2010). To that end, a mixed model ANOVA was conducted with Task (Libet Clock and Stream of Letters) and Binding Type (Action and Outcome Binding) as the within subject factors, and Age-Groups (Children, Mid-, Late-Adolescents and Adults) as between subject factors. There was a significant Binding Type main effect $[F(1,108)=23.17$,
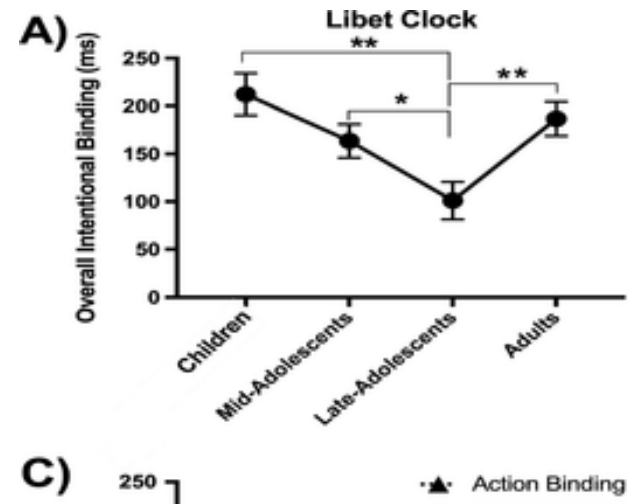

B)
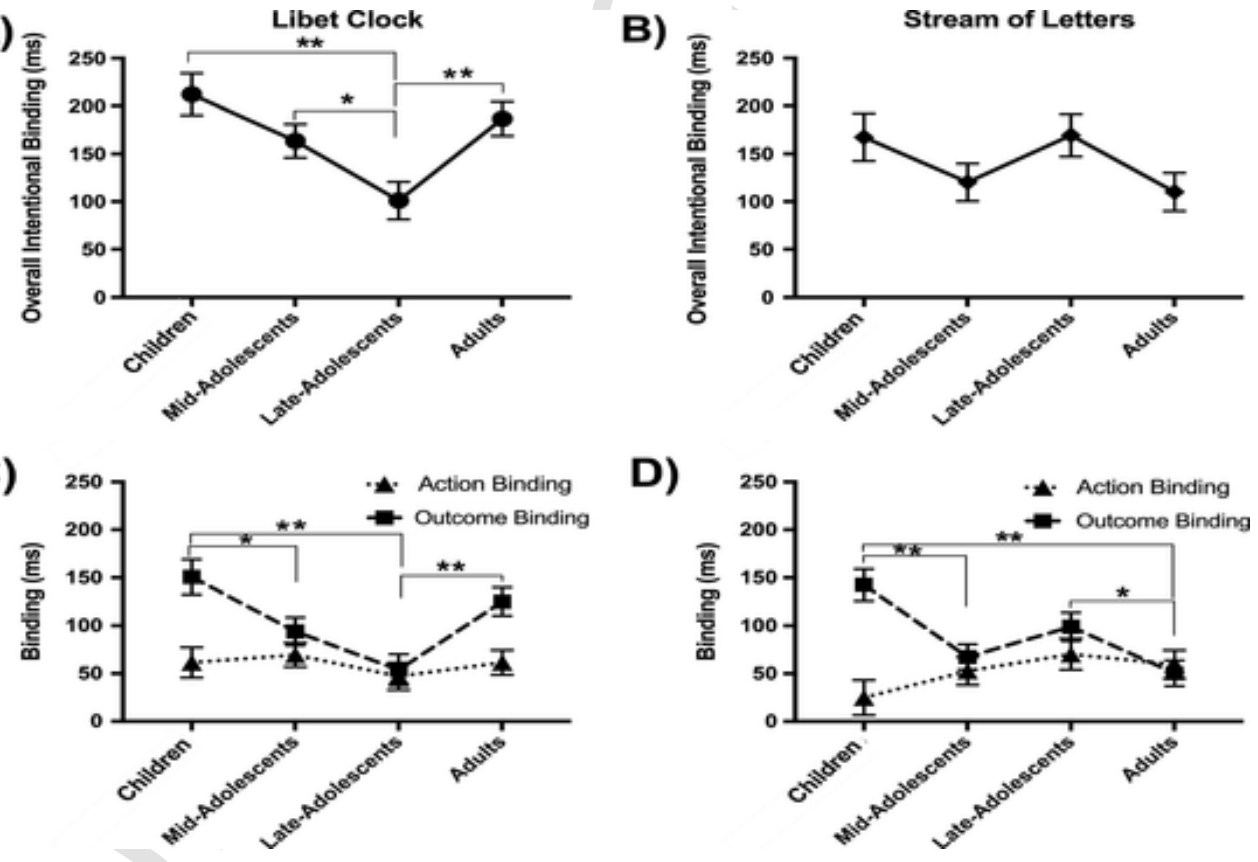

D)

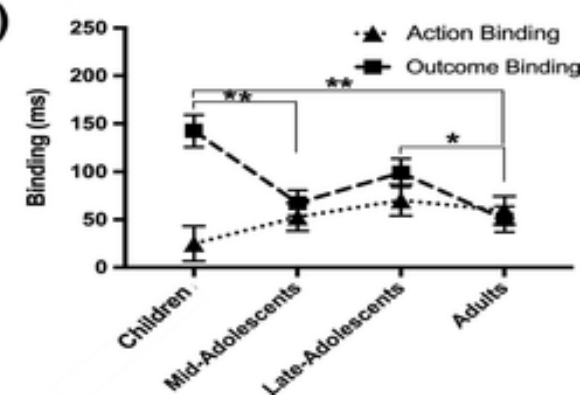

Fig. 3. Intentional binding scores measured with the Libet Clock and Stream of Letters tasks. Intentional binding represents the perceived temporal compression between action and its outcome. Action binding is the perceived shift of action towards the outcome and outcome biding is the perceived temporal shift of outcome towards action. Outcome bindings converted into positive scores for illustration purposes. (A) Mean overall intentional binding scores measured by Libet Clock. (B) Mean overall intentional binding scores measured by Stream of Letters for. (C) Mean action and outcome binding scores measured by Libet Clock. (D) Mean action and outcome binding scores measured by Stream of Letters. Error bars represent SEM. *p $<0.05,{ }^{* *} \mathrm{p}<0.005$. 
$\left.p<0.001, \eta p^{2}=0.177\right]$, two-way Binding Type X Age-Group interaction $\left[F(3,108)=4.67, p<0.005, \eta p^{2}=0.115\right]$ and three-way Binding Type X Age-Group X Task interaction $\left[F(3,108)=2.82, p=0.042, \eta p^{2}=0.73\right]$.

Our post-hoc analysis, following the significant three-way interaction, revealed that outcome binding measured with Libet Clock decreased from childhood to late-adolescence and increased back to childhood levels in adulthood $[F(3,108)=6.1, p=0.001$, $\eta p^{2}=0.145$ ] (Fig. 3C). This was a similar developmental pattern that was observed in the overall intentional binding score, suggesting that developmental differences in outcome binding were possibly driving the effect observed there. Pairwise comparisons showed that children had significantly higher outcome binding compared to mid- $[t(52)=2.43, p=0.018]$ and late-adolescents $[t(45)=3.52, p=0.001]$ whereas adults' outcome binding did not significantly differ from children $[t(51)=0.95, p=0.34]$ and mid-adolescents $[t(63)=1.65, p=0.104]$. Late-adolescents had significantly smaller outcome bindings than both adults $[t(56)=$ $-3.20, p=0.002]$ and mid-adolescents $[t(57)=-2.12, p=0.038]$. In contrast, there were no age-dependent differences in action binding scores $[F(3,108)=0.46, p=0.7]$.

Similar to Libet Clock, in Stream of Letters task, there was also significant Age-Group effect on outcome binding $[F$ $\left.(3,108)=7.08, p<0.001, \eta p^{2}=0.165\right]$, whereas action binding did not significantly change with age $[F(3,108)=1.22, p=0.3]$. As seen in Fig. 3D, there was an overall decrease of outcome binding from childhood to adulthood with a small increase during late-adolescence. Pairwise comparisons revealed that children had significantly higher outcome binding than mid-adolescents $[t(52)=3.82, p<0.001]$ and adults $[t(51)=4.14, p<0.001]$ and marginally higher outcome binding than late-adolescents $[t(45)=1.95, p=0.057]$. Adults had significantly smaller outcome binding than late-adolescents $[t(56)=-2.27, p=0.027]$. However, there were no significant differences between mid- and late-adolescents $[t(57)=-1.64, p=0.105]$ or between mid-adolescents and adults $[t(63)=0.90, p=0.37]$ in outcome binding.

\subsection{The Libet Clock and Stream of Letters tasks - A comparison}

Our findings revealed some key differences in the pattern of age-dependent changes in intentional binding as measured by the Libet Clock and Stream of Letters tasks, which have been used to measure the same phenomena while having some methodological differences (Cavazzana et al., 2014). Therefore, we next wanted to investigate whether they show convergent validity as well as to examine whether the two tasks differ with respect to the accuracy of the judgements (judgement error), consistency of the judgements (variability) and perceived difficulty of the two tasks (self-reports).

\subsubsection{Poor convergent validity}

Since the Libet Clock and Stream of Letters tasks have been used to measure intentional binding, we predicted a positive correlation in intentional binding scores measured by the two tasks. However, this was not the case ( $\mathrm{p}$ values for significance were corrected using Benjamini-Hochberg correction (Benjamini \& Hochberg, 1995). Correlational analysis on the overall intentional binding, outcome binding and action binding scores measured in the two tasks revealed no consistent significant relationship within the four age-groups tested (Table 2). A significant positive correlation was only found between overall intentional binding measured by the Libet Clock and Stream of Letters in late-adolescents $(r=0.48, \mathrm{p}=0.012, \mathrm{~N}=26)$.

\subsubsection{Internal reliability}

We next examined the internal reliability of the two tasks across age groups using split-half correlations (Goodhew and Edwards, 2019; Hedge et al., 2018; Parsons et al., 2019). We separated odd and even trials across conditions and calculated overall intentional binding scores based on these trials. Then, we investigated the correlations of overall intentional binding scores for odd and even trials within each age group. As recommended, we used Spearman-Brown corrected estimates of split-half correlations (Parsons et al., 2019), see Table 3. Overall, we found moderate to good internal reliability in the Libet clock task across all age groups tested. In contrast, the Stream of Letters task showed poor internal reliability $\left(r_{s}=-0.18\right)$ in the children group and good internal reliability for older groups. These findings suggest that Stream of Letters task might not be a reliable task for measuring intentional binding in children, and hence suggest that Libet clock task might be more reliable task with a more stable reliability estimates to examine changes in SoA from childhood to adulthood.

Table 2

Correlation between intentional binding measured by Libet Clock and Stream of Letters in each age group.

\begin{tabular}{llll}
\hline & Overall Binding & Action Binding & Outcome Binding \\
\hline Children $(\mathrm{N}=21)$ & $\mathrm{r}=-0.08, \mathrm{p}=0.72$ & $\mathrm{r}=-0.1, \mathrm{p}=0.63$ & $\mathrm{r}=0.45, \mathrm{p}=0.037$ \\
Mid-Adolescents $(\mathrm{N}=33)$ & $\mathrm{r}=0.17, \mathrm{p}=0.33$ & $\mathrm{r}=0.38, \mathrm{p}=0.028$ & $\mathrm{r}=-0.006, \mathrm{p}=0.97$ \\
Late-Adolescents $(\mathrm{N}=26)$ & $\mathrm{r}=0.48, \mathrm{p}=0.012 * 0.16, \mathrm{p}=0.42$ & $\mathrm{r}=-0.02, \mathrm{p}=0.92$ & $\mathrm{r}=0.13, \mathrm{p}=0.46$ \\
Adults $(\mathrm{N}=32)$ & $\mathrm{r}=0.23, \mathrm{p}=0.2$ & $0.38, \mathrm{p}=0.03 \quad$ \\
\hline
\end{tabular}


Table 3

Spearman-Brown corrected estimates of split half reliability for Libet Clock and Stream of Letters in each age group.

\begin{tabular}{lll}
\hline & Libet Clock & Stream of Letters \\
\hline Children $(\mathrm{N}=21)$ & $\mathrm{r}_{\mathrm{s}}=0.70$ & $\mathrm{r}_{\mathrm{s}}=-0.18$ \\
Mid-Adolescents $(\mathrm{N}=33)$ & $\mathrm{r}_{\mathrm{s}}=0.84$ & $\mathrm{r}_{\mathrm{s}}=0.84$ \\
Late-Adolescents $(\mathrm{N}=26)$ & $\mathrm{r}_{\mathrm{s}}=0.86$ & $\mathrm{r}_{\mathrm{s}}=0.88$ \\
Adults $(\mathrm{N}=32)$ & $\mathrm{r}_{\mathrm{s}}=0.89$ & $\mathrm{r}_{\mathrm{s}}=0.93$ \\
\hline
\end{tabular}

\subsubsection{Comparing judgement errors (JEs)}

To further investigate the nature of the differences observed between the two tasks we compared the Libet Clock and the Stream of Letters in terms of the accuracy of the judgements (judgement error), consistency of the judgements (variability) and perceived difficulty of the two tasks (self-reports). JEs can be negative or positive since the reported timings can be earlier or later than the real timings. Hence, the magnitude of the error quantified by absolute values of JEs were used for this analysis since the average of the negative and positive values would cancel each other out and would not represent the magnitudes of the errors. Absolute JE was calculated for each participant across conditions and tasks (Table 4). Judgement errors made in the Libet Clock and the Stream of Letters tasks were examined in the four age groups tested using a mixed model ANOVA on the absolute JE scores with Task (Libet Clock and Stream of Letters) and Condition (Baseline-Action, Baseline-Beep, Experimental-Action and Experimental-Beep) as the within-subject variables, and Age-Group (Children, Mid-Adolescents, Late-Adolescents, Adults) as a between-subject variable. There was a significant main effect of Task $\left[F(1,108)=13.29, p<0.001, \eta p^{2}=0.11\right]$ where participants showed on average $13.5 \mathrm{~ms}$ smaller JE in the Stream of Letters $(M=71, S D=2.6)$ compared to the Libet Clock $(M=84.5$, SD = 3) method (Fig. 4A). However, there was no significant interaction of Task with Age-Group or Condition ( $\mathrm{p}>0.12$ ). There was also a significant main effect of Condition $\left[F(1.9,205.88)=40.7, p<0.001, \eta p^{2}=0.274\right]$ and a condition by Age-Group interaction $[F(5.74,210.49)=2.64$, $\left.p=0.019, \eta p^{2}=0.067\right]$.

Pairwise comparisons showed that all groups had significantly smaller JE in the baseline conditions than the experimental conditions. That is, when reporting the time of action, children $[t(20)=-2.19, p=0.04]$, mid adolescents $[t(32)=-4.81, p<0.001]$, late-adolescents $[t(25)=-4.68, p<0.001]$ and adults $[t(31)=-5.39, p<0.001]$ had smaller JE in the baseline compared to the experimental condition. Likewise, when reporting the time of the beep, children $[t(20)=-2.98, p=0.007]$, mid-adolescents $[t(22)=-7.26, p<0.001]$, late-adolescents $[t(25)=-4.69, p<0.001]$ and adults $[t(31)=-4.33, p=0.001]$ had smaller JE in the baseline compared to the experimental condition. Considering age dependent changes in JE within each condition, there were no significant differences across age groups $(\mathrm{p}>0.05)$ in Baseline-Action condition. In Baseline-Beep condition, children had greater JE than mid-adolescents $[t(24.5)=4.42, p<0.001]$, late-adolescents $[t(45)=2.82, p=0.007]$ and adults $[t(51)=3.43, p=0.001]$, and other comparisons across age groups were not significant $(\mathrm{p}>0.05)$. In Experimental-Action condition, mid-adolescents had greater JE than children $[t(52)=2.92, p=0.005]$ and there were no other significant comparisons (p $>0.05)$. Finally, in Experimental-Beep condition, there were no significant JE differences across age groups $(\mathrm{p}>0.05)$. Overall, participants showed greater JEs in experimental conditions compared to baseline conditions and the age effects on JEs differed based on the condition. There was no main effect of Age Group $\left[F(3,108)=1.63, p=0.19, \eta p^{2}=0.043\right]$.

Table 4

Absolute judgement errors and standard deviations in millisecond for the Libet Clock (LC) and Stream of Letters (SoL) tasks across age groups and conditions. Standard deviations (SD) presented in brackets.

\begin{tabular}{|c|c|c|c|c|c|c|c|c|c|}
\hline & & \multicolumn{4}{|c|}{ Average Absolute Judgement Errors } & \multicolumn{4}{|c|}{ Average Standard Deviations } \\
\hline & & $\begin{array}{l}\text { Baseline } \\
\text { Action }\end{array}$ & $\begin{array}{l}\text { Exp. } \\
\text { Action }\end{array}$ & $\begin{array}{l}\text { Baseline } \\
\text { Beep }\end{array}$ & $\begin{array}{l}\text { Exp. } \\
\text { Beep }\end{array}$ & $\begin{array}{l}\text { Baseline } \\
\text { Action }\end{array}$ & $\begin{array}{l}\text { Exp. } \\
\text { Action }\end{array}$ & $\begin{array}{l}\text { Baseline } \\
\text { Beep }\end{array}$ & $\begin{array}{l}\text { Exp. } \\
\text { Beep }\end{array}$ \\
\hline \multirow{2}{*}{$\begin{array}{l}\text { Pre- } \\
\text { Adolescent }\end{array}$} & LC & $82(57)$ & $92(72)$ & $58(37)$ & $145(98)$ & 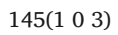 & $95(31)$ & $106(38)$ & $120(64)$ \\
\hline & SoL & $52(43)$ & $81(54)$ & $70(44)$ & $91(73)$ & $163(34)$ & $162(36)$ & $141(33)$ & $172(44)$ \\
\hline \multirow{2}{*}{$\begin{array}{l}\text { Mid- } \\
\text { Adolescent }\end{array}$} & LC & $77(60)$ & $140(82)$ & $33(25)$ & $101(70)$ & $110(79)$ & $102(54)$ & $78(21)$ & $100(43)$ \\
\hline & SoL & $70(54)$ & $121(77)$ & $25(25)$ & $73(52)$ & $122(36)$ & $132(43)$ & $88(45)$ & $129(40)$ \\
\hline \multirow{2}{*}{$\begin{array}{l}\text { Late- } \\
\text { Adolescent }\end{array}$} & LC & $57(45)$ & $94(69)$ & $51(38)$ & $85(78)$ & $92(66)$ & $82(41)$ & $80(30)$ & $102(45)$ \\
\hline & SoL & $51(46)$ & $108(83)$ & 27 (34) & $102(54)$ & 120 (39) & $119(40)$ & $67(42)$ & 125 (31) \\
\hline \multirow[t]{2}{*}{ Adult } & LC & $62(34)$ & 111 (77) & $42(35)$ & 113 (87) & 74 (29) & 78 (39) & 63 (15) & $92(40)$ \\
\hline & SoL & $58(46)$ & $100(88)$ & $28(32)$ & $73(66)$ & $108(38)$ & $113(43)$ & $56(38)$ & $106(36)$ \\
\hline
\end{tabular}




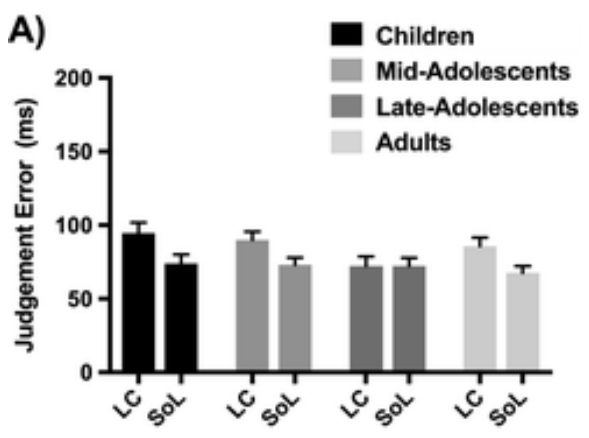

C)

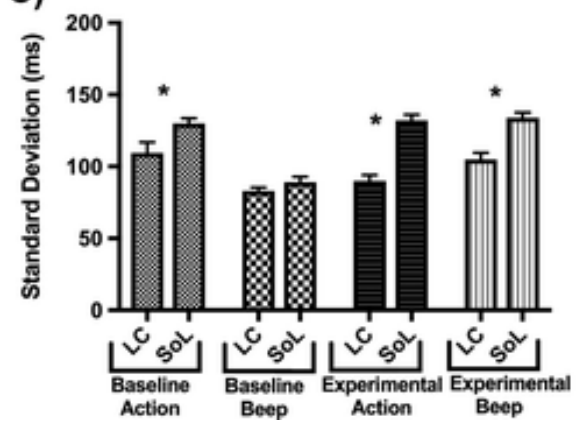

B)

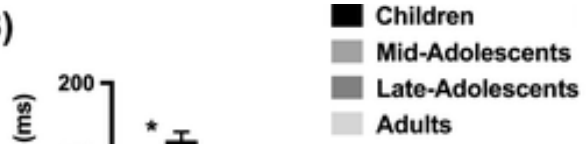

D)

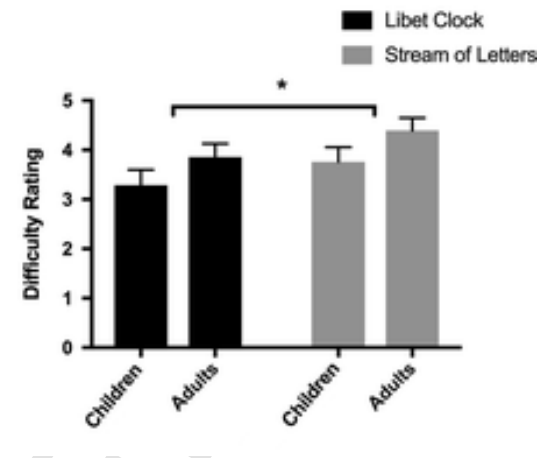

Fig. 4. (A) Overall absolute judgment errors in the Stream of Letters task was significantly less than in the Libet Clock task. (B) In contrast, significant less variability was observed in the Libet Clock compared to Stream of Letters across all four age groups as measured by the mean standard deviations of the judgement errors in each task in each age group. (C) Judgement error variability was smaller in Libet Clock compared to Stream of Letters in all conditions except for Baseline-Beep. (D) The Libet Clock was rated as less difficult than the Stream of Letters by both children (youngest age group) and adults (oldest age group), and children reported both tasks to be less difficult than adults (task difficulty was rated on a 1-7 Likert scale, with 7 being very difficult). LC, Libet Clock task; SoL, Stream of Letters task. Error bars represent SEM. *p $<0.05$.

\subsubsection{Comparing judgement error variability}

To investigate the variability of the judgement errors measured with the Libet Clock and the Stream of Letters tasks we calculated the mean standard deviation of judgment errors for each participant across conditions and tasks (Table 4). As in judgement error analysis, same mixed model ANOVA was conducted but on the average standard deviations this time. The main effect of Task $[F$ $\left.(1,108)=63.71, p<0.001, \eta p^{2}=0.371\right]$, Age-Group $\left[F(3,108)=17.34, p<0.001, \eta p^{2}=0.325\right]$, and their interaction $[F(3$, $\left.108)=2.99, p=0.034, \eta p^{2}=0.077\right]$ was significant. Pairwise comparisons showed that variability in the Libet Clock was significantly smaller compared to variability in the Stream of Letters within all Age-Groups (Fig. 4B): children [t(20) $=-5.68, p<0.001]$, mid-adolescents $[t(32)=-2.92, p=0.006]$, late-adolescents $[t(25)=-2.95, p=0.007]$ and adults $[t(31)=-4.67, p<0.001]$. Furthermore, there were age differences in terms of variability in both tasks. In the Libet Clock task, children had significantly larger variability than both late-adolescents $[t(45)=2.41, p=0.02]$ and adults $[t(51)=4.23, p<0.001]$, and adults had significantly smaller variability than mid-adolescents $[t(63)=-2.71, p=0.009]$. Similarly, in the Stream of Letters condition, children had significantly larger variability than the older age groups: mid-adolescents $[t(52)=5.46, p<0.001]$, late-adolescents [t(45) $=6.90$, $p<0.001]$ and adults $[t(51)=8.65, p<0.001]$, also adults had significantly smaller variability than mid-adolescents $[t(63)=$ $-3.19, p=0.002]$. There were no significant differences between other comparisons $(\mathrm{p}>0.05)$. Furthermore, there was a significant main effect of Condition $\left[F(2.75,297.86)=31.97, p<0.001, \eta p^{2}=0.228\right]$ and an interaction between Condition and Task $\left[F(2.82,304.52)=9.83, p<0.001, \eta p^{2}=0.083\right]$. Pairwise comparisons showed that the Libet Clock had significantly lower variability compared to the Stream of Letters in most of the conditions: Baseline-Action condition $[t(111)=-3.47, p=0.001]$, Baseline-Beep condition [ $t\left(\begin{array}{lll}1 & 1 & 1\end{array}\right)=-0.97, p=0.33$, Experimental-Action condition $\left[t\left(\begin{array}{lll}1 & 1 & 1\end{array}\right)=-8.23, p<0.001\right.$, and Experimental-Beep condition $\left[t\left(\begin{array}{lll}1 & 1 & 1\end{array}\right)=-5.95, p<0.001\right]$, and the smallest variability both in the Libet Clock and Stream of letters tasks was observed in the Baseline Beep condition (Fig. 4C).

\subsubsection{Self reports of task difficulty}

In a previous study, Cavazzana et al., (2014) proposed that the Stream of Letters task would be easier for children in comparison to the Libet Clock task. However, the difficulty of these tasks was not investigated in that study. Therefore, we investigated task difficulty reports, as measured by self-report in child and adult groups (see Methods), using a mixed model ANOVA with Task (Libet Clock and Stream of Letters) as a within subject factor and Age-Group as a between subject factor. In contrast to the previous suggestion, there was a main effect of Task where Libet Clock $(M=3.62$, SD $=0.2)$ was reported to be easier than Stream of Letters $(\mathrm{M}=4.14, \mathrm{SD}=0.18),\left[F(1,46)=5.46, p=0.024, \eta p^{2}=0.106\right]$ (Fig. 4D). There was also a significant main effect of Age- 
Group, where children $(\mathrm{M}=3.52, \mathrm{SD}=0.23)$, reported that they found the tasks to be easier in comparison to adults $(\mathrm{M}=4.24$, $\mathrm{SD}=0.2),\left[F(1,47)=5.22, p=0.027, \eta p^{2}=0.102\right]$. There was no interaction between Task and Age-Group $[F(1,46)=0.03$, $p=0.85]$.

\section{Discussion}

This study investigated the developmental trajectory of SoA as indexed by perceived action-outcome temporal linkage (intentional binding) from childhood to adulthood using the Libet Clock and Stream of Letters tasks. A significant developmental effect was found with a U-shaped developmental trajectory in the Libet Clock task where children and adults showed greater intentional binding, but it was reduced starting from mid-adolescence and reached its minimum during late-adolescence. This suggests adolescents might be experiencing reduced agency over their actions and the consequences of these actions. In contrast, no age group differences were observed in intentional binding as measured by Stream of Letters task. Notably, however, follow up analysis of action and outcome binding suggested that the effects observed in intentional binding scores were driven by age-specific significant changes in outcome binding measured by both tasks. An assessment of the convergent validity of these tasks showed that they have poor correlations across age groups suggesting they might be measuring different aspects of intentional binding. Further comparisons of the tasks revealed that participants' timing judgements were smaller on average in the Stream of Letters, but their judgements were more stable (smaller variability) in the Libet Clock task. Consistent with this, children and adults reported that performing the Libet Clock was easier than Stream of Letters. These findings suggest that the Libet Clock might be an easier and a more reliable method for investigating intentional binding within the age range of this study (9-28 years old). The developmental trajectories and task differences observed in this study may have important implications for future work aiming to understand the development of SoA and SoA related neurodevelopmental disorders as well as some age-specific behaviours such as increased risk-taking during adolescence.

\subsection{Developmental trajectory of intentional binding and its relationship to brain maturation and cognitive processes}

We found that intentional binding measured by the Libet Clock task decreased from childhood to late-adolescence and increased back to children levels in adulthood. This trajectory suggests an adolescent-specific developmental change in implicit SoA with an exaggerated agency experience in children and adults and conversely a decreased agency experience during adolescence. Adolescence is a critical developmental period where the brain undergoes a substantial remodelling process specifically underlined by maturational changes in the frontal areas together with grey matter decrease and white matter increase which improves the efficiency and the connectivity of the system (Asato et al., 2010; Luna et al., 2001; Mills et al., 2016; Sowell et al., 2003, 1999; Zhou et al., 2015). The maturational changes of the brain regions including SoA related areas such as pre-frontal/ frontal (DLPFC and pre-SMA/ SMA), parietal (angular gyrus) cortex and striatum might be underlying the observed reduction of the subjective temporal linkage between actions and outcomes during adolescence. This might result in more realistic action outcome temporal relationship compared to children. That is because both mid- and late-adolescents showed smaller intentional binding effect reflecting that the perceived temporal gap between action and outcome was closer to the actual temporal gap introduced between action and the outcome in the experiment (250 ms). One would expect this effect to stabilise from late-adolescence to adulthood or continue to improve until adulthood with similar or even less intentional binding in adults. However, this was not the case, using the Libet clock task we found that intentional binding in adults increased back to approximately to the levels observed in childhood.

One possible explanation of the developmental rebound effect observed in adults might be related to the shifts in cognitive processes in relation with brain maturation. It has been previously suggested that a balanced and context dependent combination of retrospective and predictive processes would be necessary for constructing a reliable SoA (Chambon, Sidarus, \& Haggard, 2014; Haggard, 2017; Moore \& Fletcher, 2012; Synofzik et al., 2013). Retrospective processes are associated with external cues that take place after action and outcome occurrence, and are involved in inferring a causal relationship between intentions and actions retrospectively based on the sensory evidence collected (retrospective explanation of what has happened based on external cues) (Chambon et al., 2014; Haggard, 2017; Wegner, 2002; Wegner and Wheatley, 1999). On the other hand, predictive processes result from internal cues that take place before the action is performed such as intention, action preparation and predicting outcomes and involved in the construction of agency experience prospectively (Chambon et al., 2014; Waszak et al., 2012; Yoshie \& Haggard, 2017). And, depending on the context (predictable vs. unpredictable), a balanced combination of these processes would be required for constructing a reliable sense of agency.

The U shaped developmental trajectory we found might reflect a developmental shift from relying on retrospective to predictive processes from childhood to adulthood with a balance occurring during adolescence. It is possible that we observed retrospective over-binding in childhood and predictive over-binding in adulthood but a more balanced combination of these in adolescence. Hence, children might have based their SoA on retrospective processes; in other words, they "explained away" what just happened based on the sensory evidence collected since their predictive processes might not yet be reliable enough due to the much later maturation of SoA related brain regions (fronto-parietal network). On the other hand, adults may have based their SoA on predictive processes where they "predicted away" what is going to happen based on the strong learned priors about the action outcomes. Hence, both of these scenarios would result in high intentional binding associated with exaggerated agency. In contrast, adolescents might have based their SoA on a balanced combination of predictive and retrospective processes which can help them to have an experience of agency that is reflected by an action-outcome temporal linkage that is closer to the reality (closer to the actual tempo- 
ral gap inserted between actions and outcomes). If this is the case, this whole process can be viewed as a developmental transition from using external cues to using internal cues when constructing the experience of agency. The balance between these processes during adolescence might be beneficial to refine the predictive processes and reduce the errors that might occur in adulthood. This hypothesis needs to be tested in the future studies possibly using a similar experimental design to Moore and Haggard, (2008) where they manipulated the probability of the outcome occurrence or using EEG to isolate these processes such as recording the brain activity associated with the predictive processes including readiness potential (Jo et al., 2014) and auditory N1 attenuation (Waszak et al., 2012).

Interestingly, the developmental trajectory we observed in overall intentional binding seems to be a result of age-specific changes in outcome binding, but not action binding, an effect observed in both the Libet Clock and Stream of Letters tasks. We found that outcome binding followed the similar developmental trajectory with overall intentional binding. In contrast, there were no significant changes in action binding in the transition from childhood to adulthood. The differential effects of age on action and outcome binding suggest that the mechanisms regulating these processes might follow different developmental trajectories. Action and outcome binding have been suggested to be mediated by different mechanisms previously. That is outcome binding is more associated with predictive processes (Waszak et al., 2012) whereas action binding is associated with both retrospective and predictive processes (Moore \& Haggard, 2008). Hence, it is possible that the lack of age effect on action binding might reflect earlier maturation of retrospective processes whereas prolonged changes occurring in the outcome binding reflects more of a later maturation in the predictive processes. Predictive processes' involvement in outcome but not action binding was suggested by brain stimulation and neuroimaging studies suggesting the involvement of pre-SMA, an area related to action initiation and generating efferent copy (Moore et al., 2010; Jo et al., 2014), and angular gyrus, an area thought to be associated with both action and outcome monitoring (Khalighinejad \& Haggard, 2015). Therefore, taken together, the prolonged developmental changes we observed in outcome binding might be related to the slow maturational changes in fronto-parietal network that is involved in these processes.

\subsection{Developmental trajectory of implicit agency is consistent with explicit studies}

Our finding that children show increased levels of intentional binding associated with increased SoA is consistent with other studies focusing on the metacognition of agency. They found that children show increased SoA especially for the artificial performance increase (Metcalfe, Eich, \& Castel, 2010) and for positive action outcomes (Van Elk et al., 2015) suggesting an increased self-agency bias in children compared to adults. It should be noted here that their adult group ( $M=20$ years-old in Van Elk et al. (2015) and between 18 and 24 years old in Metcalfe et al. (2010)) corresponds approximately to our late-adolescent age group (age range: 18-20). Hence, the decreasing levels of intentional binding up to late-adolescence seems to be in line with decreased judgement of agency observed in these studies.

However, our findings are not consistent with the study of Cavazzana et al (2014), an intentional binding study which also used the Stream of Letters task. They found that children showed a relatively diminished intentional binding effect mediated by a reduction in action binding compared to adults suggesting a reduced SoA for children. In contrast, we found a lack of age effect on overall intentional binding and a significant age effect on outcome binding but not action binding using the Stream of Letters task. Although we followed a similar procedure, there was a key difference between Cavazzana et al., (2014) and the study presented here. Differently from the current study, they included additional passive action condition where an apparatus was pressing participants' fingers in order to create the button press action passively. It is possible that this condition might have increased children's awareness about the fact that they might not be the agent all the time, whereas adults might not have been influenced by this contextual effect. Although this might explain the differential findings between this and Cavazzana et al. (2014) studies, further investigation is needed.

In addition to these, there have been other studies examining the childhood period using interval estimation method for measuring binding effect (Blakey et al., 2019; Lorimer et al., 2020). It was shown that binding phenomenon can be observed as young as 4-5 $(\mathrm{M}=4.5)$ years old and its magnitude did not change significantly throughout childhood until 10-11 ( $\mathrm{M}=10.5)$ years old (Blakey et al., 2019). In addition to this, another study compared adults ( $M=23$ years old) to children (four age groups: 6 - 7; 7 - 8; 8 - 9; 9 - 10) and found that intentional binding did not significantly change throughout childhood and compared to adulthood (Lorimer et al., 2020). Even though methodologies are different between current study (mental chronometry) and Lorimer et al. (2020) (interval estimation), our finding that children (9-10) have similar intentional binding with adults (25-28) are consistent. In addition to these studies, current study added adolescent period which was not investigated in these studies and suggested that adolescence might be a critical period for intentional binding development.

\subsection{A comparison of the Libet Clock and Stream of Letters tasks}

Our comparisons between the Libet Clock and the Stream of Letters tasks revealed that although the Stream of Letters resulted in on average approximately $14 \mathrm{~ms}$ closer judgements to the real timings of the events, Libet Clock might be more reliable when judging the time of the events since participants show smaller variability in the size of their judgment error in all age groups and in most of the task conditions, and it might be easier as reported by children and adults. A less variable measure would be beneficial in intentional binding tasks than smaller judgement errors. This is because baseline correction (subtracting judgement errors in baseline conditions from experimental conditions) in intentional binding tasks would eliminate the judgement error differences in terms of measuring the intentional binding effect but higher variability of the measurement would not be compensated. Further- 
more, based on our split-half internal reliability estimates, we found that Stream of Letters task might not be a reliable task for measuring intentional binding in children, although reliable for older age groups, and Libet clock task might be more reliable task with a more stable reliability estimates across age groups from childhood to adulthood. Based on these results and the more frequent use of the Libet Clock in the literature that can elicit comparisons between studies, we suggest Libet Clock might be preferable task to use when measuring intentional binding within the age range of this study.

Critically, we found very little evidence of convergent validity between the Libet Clock and Stream of Letters tasks. Furthermore, developmental effects in intentional binding measured with Stream of Letters and Libet Clock tasks were task-dependent. In the Stream of Letters task no age differences were found in the overall intentional binding scores, while clear age-effects were found in the Libet clock task, with a U-shaped developmental trajectory. Likewise, while both tasks showed significant developmental effects in outcome binding, the trajectory showed differences between tasks especially for adults. Taken together, these findings and poor convergent validity might point to deeper problems when measuring intentional binding. Intentional binding has been shown to be sensitive to the variables that also affect sense of agency such as voluntariness and temporal contiguity (Haggard et al., 2002; for review, Moore \& Obhi, 2012; Imaizumi and Tanno, 2019) and the relationship between intentional binding and explicit sense of agency has recently been shown (Imaizumi and Tanno, 2019). However, it is possible that tasks that have been used to measure intentional binding might be measuring different features based on the task specifications. Indeed, our findings suggest that this might be the case and these tasks might be tapping into different aspects of intentional binding. This could be due to the key difference in predictability between these two tasks. In the Libet Clock task, the clock hand motion is predictable in terms of where it would move in time but the quickly changing random letters are unpredictable. This predictability issue could have affected what each task might be measuring in two ways. First, the Libet Clock might be easier due to this. Indeed, our participants (children and adult) reported that they found the Libet Clock to be easier than the Stream of Letters, a finding supported by the smaller variability in judgement errors in the Libet Clock task. Second, the predictability of each task might have created a contextual effect, and this might be the main reason why we found some differential developmental trajectories as measured by these two tasks. For example, in adults, we found high outcome binding effect when measured with Libet Clock, but we found low outcome binding effect when measured with Stream of Letters. Adults might have shown predictive over-binding when measured with the Libet Clock; it is also possible that they did not show this predictive over-binding when there is a contextual unpredictability as seen in the Stream of Letters. The effect of measurement method's predictability on intentional binding has not been investigated previously but we suggest it might have effects as observed in the current study.

\section{Study limitations and future directions}

The results from the current study need to be considered in light of this study's limitations. First, we used an implicit measure, intentional binding, to quantify the SoA. However, SoA has been proposed to encompass two levels. That is, feeling of agency which is the background low-level feeling of being in charge of our actions (implicit), and judgement of agency which is the high-level experience of agency that arises when we make conscious evaluations of agency (explicit) (Synofzik, Vosgerau, \& Newen, 2008). Intentional binding reflects the lower-level feeling of agency (Moore \& Obhi, 2012). Therefore, the developmental trajectory found in this study might not reflect the developmental trajectory of the judgements (metacognition) of agency. Second, this study was not designed to address differential processes underlying intentional binding such as predictive and retrospective processes. Therefore, our suggestion about a possible developmental shift from relying on retrospective to predictive processes in the transition from childhood to adulthood, needs to be tested in future studies that isolate these processes. Third, we only compared two mental chronometry methods of measuring intentional binding. Other methods such as interval estimation (for example, Engbert et al., 2007) might have some advantages such as focusing on the interval in between action and outcome instead of focusing on isolated timings of the events as seen in mental chronometry methods. However, interval estimation methods do not allow isolating action and outcome binding, which we found to show different developmental trajectory. Fourth, the higher variability observed in the Stream of Letters task might be a result of this task's poorer temporal precision (a letter represents $150 \mathrm{~ms}$ ) compared to Libet Clock task (a clock position represents $42 \mathrm{~ms}$ ). However, the reason behind the variability difference across tasks in our experiment is not clear, since it could also be caused by the predictability of the tasks (e.g., participants might be giving more consistent answers in a more predictable task, the Libet clock). Hence, future studies need to investigate the effects of predictability and temporal precision of the tasks on intentional binding. Finally, the auditory stimulus used as the outcome did not have any specific value for the participants. Therefore, the developmental trajectory observed might be different when socially relevant, emotional or rewarding stimuli are used. This is especially important for adolescence where we see an increased responsivity to both rewarding and aversive stimulus (Casey, 2015). Hence, developmental trajectory of SoA when the outcomes of actions have value, we suggest, is an important direction for future research.

Future studies might also want to focus on the functional significance of the developmental effects observed in this study. For instance, adolescence is associated with an increase in risk-taking behaviour which can help them to explore their environment but can also result in harm to self and others (Casey et al., 2010; Paus, Keshavan, \& Giedd, 2008; Kim-Spoon et al., 2016; Steinberg, 2008). It is possible that the reduced implicit control over the outcomes of their actions during mid- and late-adolescence might be a partial underlying factor for the increased risk-taking behaviour since they might feel less responsible for the consequences of their risky actions, an idea which requires further exploration. In addition, adolescence is also linked with increased vulnerability for developing psychotic disorders such as schizophrenia (Gomes et al., 2017; Harrop \& Trower, 2001), a disorder associated with impairments in SoA (e.g. control dellusions) (Frith et al., 2000a; Synofzik et al., 2010; Voss et al., 2010). It has been shown previ- 
ously that schizophrenia patients show retrospective over-binding with a specific impairment in predicting the action outcomes (Voss et al., 2010). This could be associated with the lack of a possible developmental shift from retrospective to predictive over-binding from childhood to adulthood, where normally this critical shift might occur during adolescence as proposed in this study. Another idea that can be explored in the future studies. Finally, increased temporal association during childhood might be beneficial for children's learning of action outcome causal relationships and it has been reported to be grounded in the causal learning early on since binding effect can be observed in as young as 4 years old children (Blakey et al., 2019).

\section{Conclusions}

We found significant age-differences in SoA indexed by intentional binding. Interestingly, observed developmental trajectories were driven by age specific differences in outcome binding in both tasks. Children and adults, showed higher outcome binding, whereas adolescents showed significant reduction in outcome binding, with lowest levels observed during late-adolescence in the Libet Clock task. In contrast, in the Stream of Letters task, outcome binding remained low in the adult group, which suggests that observed developmental changes might be task sensitive. Based on our comparison of the Libet Clock and Stream of Letters tasks, Libet Clock might be easier and more reliable task for measuring intentional binding in the age range of this study (9-28). Notably, the age-dependent changes we found for intentional binding during adolescence, do suggest an adolescent-specific reduction in the implicit feeling that they are in charge of their own actions, and that adolescence might be a unique developmental period for SoA. We suggest that the prolonged U-shaped developmental trajectory that we observed in the Libet Clock task might be mediated by a developmental shift in the cognitive strategies used from retrospective inference to predictive construction of agency experiences. Observed reduction in the implicit agency during adolescence might be associated with the increased vulnerability of developing psychotic disorders during adolescence resulting from an impairment in SoA (e.g. control delusions), as well as some adolescence-specific behaviours such as increased risk-taking as they might experience a reduction in the association between risky behaviours and their harmful consequences.

\section{Uncited references}

\section{CRediT authorship contribution statement}

Ali Aytemur: Conceptualization, Methodology, Software, Formal analysis, Investigation, Data curation, Writing - original draft, Visualization. Liat Levita: Conceptualization, Methodology, Writing - review \& editing, Supervision.

\section{Acknowledgements}

This research did not receive any specific grant from funding agencies in the public, commercial, or not-profit sectors. We are thankful to Dr. Robert Schmidt for his helpful comments on the manuscript.

\section{References}

Alexander, G.E., \& Crutcher, M.D. (1990). Functional architecture of basal ganglia circuits: Neural substrated of parallel processing. Trends in Neuroscience, 13(7), 266-271. doi:10.1016/0166-2236(90)90107-L.

Asato, M.R., Terwilliger, R., Woo, J., \& Luna, B. (2010). White Matter Development in Adolescence: A DTI Study. Cerebral Cortex, 20(9), 2122-2131. doi:10.1093/ cercor/bhp282.

Benjamini, Yoav, \& Hochberg, Yosef (1995). Controlling the false discovery rate: a practical and powerful approach to multiple testing.. Journal of the Royal statistical society: series B (Methodological), 57(1), 289-300. doi:https://doi.org/10.2307/2346101.

Blakey, E., Tecwyn, E.C., McCormack, T., Lagnado, D.A., Hoerl, C., Lorimer, S., \& Buehner, M.J. (2019). When causality shapes the experience of time: Evidence for temporal binding in young children. Developmental Science, 22(3). doi:10.1111/desc.12769.

Casey, B., Getz, S., \& Galvan, A. (2008). The adolecent brain. Developmental Review, 28(1), 62-77. doi:10.1016/j.dr.2007.08.003.The.

Casey, B.J. (2015). Beyond Simple Models of Self-Control to Circuit-Based Accounts of Adolescent Behavior. Annu. Rev. Psychol., 66(1), 295-319. doi:10.1146/ annurev-psych-010814-015156.

Casey, B.J., Jones, R.M., Levita, L., Libby, V., Pattwell, S.S., Ruberry, E.J., ... Somerville, L.H. (2010). The storm and stress of adolescence: Insights from human imaging and mouse genetics. Dev. Psychobiol.. doi:10.1002/dev.20447.

Cavazzana, A., Begliomini, C., \& Bisiacchi, P.S. (August 2014). Intentional binding effect in children: Insights from a new paradigm. Frontiers in Human Neuroscience, 8, 1-11. doi:10.3389/fnhum.2014.00651.

Chambon, V., Sidarus, N., \& Haggard, P. (May 2014). From action intentions to action effects: How does the sense of agency come about? Frontiers in Human Neuroscience, 8, 1-9. doi:10.3389/fnhum.2014.00320.

Ebert, J.P., \& Wegner, D.M. (2010). Time warp: Authorship shapes the perceived timing of actions and events. Consciousness and Cognition, 19(1), 481-489. doi:10.1016/j.concog.2009.10.002.

Engbert, K., Wohlschläger, A., Thomas, R., \& Haggard, P. (2007). Agency, subjective time, and other minds. Journal of Experimental Psychology: Human Perception and Performance, 33(6), 1261-1268. doi:10.1037/0096-1523.33.6.1261.

Ernst, M. (2014). The triadic model perspective for the study of adolescent motivated behavior. Brain and Cognition, 89, 104-111. doi:10.1016/j.bandc.2014.01.006.

Frith, C.D. (2014). Action, agency and responsibility. Neuropsychologia, 55(1), 137-142. doi:10.1016/j.neuropsychologia.2013.09.007.

Frith, C. D., Blakemore, S. J., \& Wolpert, D. M. (2000). Abnormalities in the awareness and control of action. Discovering the Social Mind: Selected Works of Christopher D. Frith, (March), 64-100. https://doi.org/10.4324/9781315630502.

Frith, C.D., Blakemore, S.J., \& Wolpert, D.M. (2000). Explaining the symptoms of schizophrenia: Abnormalities in the awareness of action. Brain Research Reviews, 31(2-3), 357-363. doi:10.1016/S0165-0173(99)00052-1.

Gallagher, S. (2000). Philosophical conceptions of the self: Implications for cognitive science. Trends in Cognitive Sciences, 4(1), 14-21. doi:10.1016/ S1364-6613(99)01417-5. 
Gogtay, N., Giedd, J.N., Lusk, L., Hayashi, K.M., Greenstein, D., Vaituzis, A.C., ... Thompson, P.M. (2004). Dynamic mapping of human cortical development during childhood through early adulthood. Proceedings of the National Academy of Sciences, 101(21), 8174-8179. doi:10.1073/pnas.0402680101.

Gomes, F.V., Rincon-Cortes, M., \& Grace, A.A. (2017). Adolescence as a period of vulnerability and intervention in schizophrenia: Insights from the MAM Model. Neuroscience and Biobehavioral Reviews, 70, 260-270. doi:10.1016/j.neubiorev.2016.05.030.Adolescence.

Goodhew, S.C., \& Edwards, M. (2019). Translating experimental paradigms into individual-differences research: Contributions, challenges, and practical recommendations. Consciousness and Cognition, 69, 14-25. doi:10.1016/j.concog.2019.01.008.

Haggard, P. (2005). Conscious intention and motor cognition. Trends in Cognitive Sciences, 9(6), 290-295. doi:10.1016/j.tics.2005.04.012.

Haggard, P. (2017). Sense of agency in the human brain. Nat Rev Neurosci, 18(4), 196-207. doi:10.1038/nrn.2017.14.

Haggard, P., Clark, S., \& Kalogeras, J. (2002). Voluntary action and conscious awareness. Nature Neuroscience, 5(4), 382-385. doi:10.1038/nn827.

Haggard, P., \& Tsakiris, M. (2009). The Experience of Agency: Feelings, Judgments, and Responsibility. Current Directions in Psychological Science, 18(4), 242-246. doi:10.1111/j.1467-8721.2009.01644.x.

Haggard, P., \& Whitford, B. (2004). Supplementary motor area provides an efferent signal for sensory suppression. Cognitive Brain Research, 19(1), 52-58. doi:10.1016/ j.cogbrainres.2003.10.018.

Harrop, C., \& Trower, P. (2001). Why does schizophrenia develop at late adolescence? Clinical Psychology Review, 21(2), 241-265. doi:10.1016/ S0272-7358(99)00047-1.

Hedge, C., Powell, G., \& Sumner, P. (2018). The reliability paradox: Why robust cognitive tasks do not produce reliable individual differences. Behav Res, 50(3), 1166-1186. doi:10.3758/s13428-017-0935-1.

Imaizumi, Shu, \& Yoshihiko, Tanno (2019). Intentional binding coincides with explicit sense of agency. Consciousness and Cognition, 67, 1-15. doi:https://doi.org/ 10.1016/J.CONCOG.2018.11.005.

Jo, H.-G., Wittmann, M., Hinterberger, T., \& Schmidt, S. (June 2014). The readiness potential reflects intentional binding. Frontiers in Human Neuroscience, 8, 1-9. doi:10.3389/fnhum.2014.00421.

Khalighinejad, N., Di Costa, S., \& Haggard, P. (2016). Endogenous Action Selection Processes in Dorsolateral Prefrontal Cortex Contribute to Sense of Agency: A Meta-Analysis of tDCS Studies of "Intentional Binding". Brain Stimulation, 9(3), 372-379. doi:10.1016/j.brs.2016.01.005.

Khalighinejad, N., \& Haggard, P. (2015). Modulating human sense of agency with non-invasive brain stimulation. Cortex, 69, 93-103. doi:10.1016/j.cortex.2015.04.015.

Kleiner, M., Brainard, D., Pelli, D., Ingling, A., Murray, R., \& Broussard, C. (2007). What's new in Psychtoolbox-3? A free cross-platform toolkit for Psychophysics with Matlab \& GNU/Octave Retrieved from. Perception, 36(14). http://www.kyb.mpg.de/fileadmin/user_upload/files/publications/attachments/ECVP2007-Kleinerslides_5490\%5B0\%5D.pdf.

Kim-Spoon, J., Kahn, R., Deater-Deckard, K., Chiu, P., Steinberg, L., \& King-Casas, B. (2016). Risky decision making in a laboratory driving task is associated with health risk behaviors during late adolescence but not adulthood. International Journal of Behavioral Development, 40(1), 58-63. doi:10.1177/0165025415577825.

Libet, B., Gleason, C. A., Wright, E. W., \& Pearl, D. K. (1983). Time of Conscious Intention To Act in Relation To Onset of Cerebral Activity (Readiness-Potential). Brain, 106(3), 623-642. https://doi.org/10.1093/brain/106.3.623.

Lorimer, S., McCormack, T., Blakey, E., Lagnado, D.A., Hoerl, C., Tecwyn, E.C., \& Buehner, M.J. (2020). The developmental profile of temporal binding: From childhood to adulthood. Quarterly Journal of Experimental Psychology, 73(10), 1575-1586. doi:10.1177/1747021820925075.

Luna, B., Thulborn, K.R., Munoz, D.P., Merriam, E.P., Garver, K.E., Minshew, N.J., ... Sweeney, J.A. (2001). Maturation of Widely Distributed Brain Function Subserves Cognitive Development. NeuroImage, 13(5), 786-793. doi:10.1006/nimg.2000.0743.

Metcalfe, J., Eich, T.S., \& Castel, A.D. (2010). Metacognition of agency across the lifespan. Cognition, 116(2), 267-282. doi:10.1016/j.cognition.2010.05.009.

Mills, K.L., Goddings, A.-L., Clasen, L.S., Giedd, J.N., \& Blakemore, S.-J. (2014). The Developmental Mismatch in Structural Brain Maturation during Adolescence. Developmental Neuroscience, 36(3-4), 147-160. doi:10.1159/000362328.

Mills, K.L., Goddings, A.-L., Herting, M.M., Meuwese, R., Blakemore, S.-J., Crone, E.A., ... Tamnes, C.K. (2016). Structural brain development between childhood and adulthood: Convergence across four longitudinal samples. NeuroImage, 141, 273-281. doi:10.1016/j.neuroimage.2016.07.044.

Moore, J., \& Haggard, P. (2008). Awareness of action: Inference and prediction. Consciousness and Cognition, 17(1), 136-144. doi:10.1016/j.concog.2006.12.004.

Moore, J.W., \& Fletcher, P.C. (2012). Sense of agency in health and disease: A review of cue integration approaches. Consciousness and Cognition, 21(1), 59-68. doi:10.1016/j.concog.2011.08.010.

Moore, J.W., \& Obhi, S.S. (2012). Intentional binding and the sense of agency: A review. Consciousness and Cognition, 21(1), 546-561. doi:10.1016/ j.concog.2011.12.002.

Moore, J.W., Ruge, D., Wenke, D., Rothwell, J., \& Haggard, P. (2010). Disrupting the experience of control in the human brain: Pre-supplementary motor area contributes to the sense of agency. Proceedings of the Royal Society B: Biological Sciences, 277(1693), 2503-2509. doi:10.1098/rspb.2010.0404.

Moretto, G., Walsh, E., \& Haggard, P. (2011). Experience of agency and sense of responsibility. Consciousness and Cognition, 20(4), 1847-1854. doi:10.1016/ j.concog.2011.08.014.

Mutlu, A.K., Schneider, M., Debbané, M., Badoud, D., Eliez, S., \& Schaer, M. (2013). Sex differences in thickness, and folding developments throughout the cortex. NeuroImage, 82, 200-207. doi:10.1016/j.neuroimage.2013.05.076.

Nagy, Z., Westerberg, H., \& Klingberg, T. (2004). Maturation of White Matter is Associated with the Development of Cognitive Functions during Childhood. Journal of Cognitive Neuroscience, 16(7), 1227-1233. doi:10.1162/0898929041920441.

Pacherie, E. (2008). The phenomenology of action: A conceptual framework. Cognition, 107(1), 179-217. doi:10.1016/j.cognition.2007.09.003.

Paus, T., Keshavan, M., \& Giedd, J.N. (2008). Why do many psychiatric disorders emerge during adolescence? Nature Reviews Neuroscience, 9(12), 947-957. doi:10.1038/nrn2513.

Parsons, S., Kruijt, A.-W., \& Fox, E. (2019). Psychological Science Needs a Standard Practice of Reporting the Reliability of Cognitive-Behavioral Measurements. Advances in Methods and Practices in Psychological Science, 2(4), 378-395. doi:10.1177/2515245919879695.

Sawyer, S.M., Azzopardi, P.S., Wickremarathne, D., \& Patton, G.C. (2018). The age of adolescence. The Lancet Child \& Adolescent Health, 2(3), 223-228. doi:10.1016/ S2352-4642(18)30022-1.

Schubert, T.W., D'Ausilio, A., \& Canto, R. (2013). Using Arduino microcontroller boards to measure response latencies. Behavior Research Methods, 45(4), 1332-1346. doi:10.3758/s13428-013-0336-z.

Sowell, E.R., Peterson, B.S., Thompson, P.M., Welcome, S.E., Henkenius, A.L., \& Toga, A.W. (2003). Mapping cortical change across the human life span. Nature Neuroscience, 6(3), 309-315. doi:10.1038/nn1008.

Sowell, E.R., Thompson, P.M., Holmes, C.J., Batth, R., Jernigan, T.L., \& Toga, A.W. (1999). Localizing Age-Related Changes in Brain Structure between Childhood and Adolescence Using Statistical Parametric Mapping. NeuroImage, 9(6), 587-597. doi:10.1006/nimg.1999.0436.

Sowell, E.R., Thompson, P.M., Holmes, C.J., Jernigan, T.L., \& Toga, A.W. (1999). In vivo evidence for post-adolescent brain maturation in frontal and striatal regions. Nature Neuroscience, 2(10), 859-861. doi:10.1038/13154.

Sowell, E.R., Thompson, P.M., Tessner, K.D., \& Toga, A.W. (2001). Mapping Continued Brain Growth and Gray Matter Density Reduction in Dorsal Frontal Cortex: Inverse Relationships during Postadolescent Brain Maturation. Journal of Neuroscience, 21(22), 8819-8829.

Sperduti, M., Delaveau, P., Fossati, P., \& Nadel, J. (2011). Different brain structures related to self- and external-agency attribution: A brief review and meta-analysis. Brain Structure and Function, 216(2), 151-157. doi:10.1007/s00429-010-0298-1.

Steinberg, L. (2008). Neuroscience Perspective on Adolescent Risk Taking. Dev. Rev., 28(1), 1-27. doi:10.1016/j.dr.2007.08.002.A.

Steinberg, L., Albert, D., Cauffman, E., Banich, M., Graham, S., \& Woolard, J. (2008). Age differences in sensation seeking and impulsivity as indexed by behavior and self-report: Evidence for a dual systems model. Developmental Psychology, 44(6), 1764-1778. doi:10.1037/a0012955.

Synofzik, M., Thier, P., Leube, D.T., Schlotterbeck, P., \& Lindner, A. (2010). Misattributions of agency in schizophrenia are based on imprecise predictions about the sensory consequences of one's actions. Brain, 262-271. doi:10.1093/brain/awp291.

Synofzik, M., Vosgerau, G., \& Newen, A. (2008). Beyond the comparator model: A multifactorial two-step account of agency. Consciousness and Cognition, 17(1), 219-239. doi:10.1016/j.concog.2007.03.010.

Synofzik, M., Vosgerau, G., \& Voss, M. (March 2013). The experience of agency: An interplay between prediction and postdiction. Frontiers in Psychology, 4, 1-8. doi:10.3389/fpsyg.2013.00127. 
van Elk, M., Rutjens, B.T., \& van der Pligt, J. (2015). The development of the illusion of control and sense of agency in 7- to-12-year old children and adults. Cognition, 145, 1-12. doi:10.1016/j.cognition.2015.08.004.

Voss, M., Chambon, V., Wenke, D., Kühn, S., \& Haggard, P. (2017). In and out of control: Brain mechanisms linking fluency of action selection to self-agency in patients with schizophrenia. Brain, 140(8), 2226-2239. doi:10.1093/brain/awx136.

Voss, M., Moore, J., Hauser, M., Gallinat, J., Heinz, A., \& Haggard, P. (2010). Altered awareness of action in schizophrenia: A specific deficit in predicting action consequences. Brain, 133(10), 3104-3112. doi:10.1093/brain/awq152.

Waszak, F., Cardoso-Leite, P., \& Hughes, G. (2012). Action effect anticipation: Neurophysiological basis and functional consequences. Neuroscience \& Biobehavioral Reviews, 36(2), 943-959. doi:10.1016/j.neubiorev.2011.11.004.

Wegner, D.M. (2002). The Illusion of Conscious Will. The MIT Press.

Wegner, D.M., \& Wheatley, T. (1999). Apparent mental causation: Sources of the experience of will.. American Psychologist, 54(7), 480-492. doi:10.1037/ 0003-066X.54.7.480.

Wolpe, N., Haggard, P., Siebner, H.R., \& Rowe, J.B. (2013). Cue integration and the perception of action in intentional binding. Experimental Brain Research, 229(3), 467-474. doi:10.1007/s00221-013-3419-2.

Wolpe, N., Moore, J.W., Rae, C.L., Rittman, T., Altena, E., Haggard, P., \& Rowe, J.B. (2014). The medial frontal-prefrontal network for altered awareness and control of action in corticobasal syndrome. Brain, 137(1), 208-220. doi:10.1093/brain/awt302.

Wolpert, D., Ghahramani, Z., \& Jordan, M. (1995). An internal model for sensorimotor integration. Science, 269(5232), 1880-1882. doi:10.1126/science:7569931.

Yoshie, M., \& Haggard, P. (2017). Effects of emotional valence on sense of agency require a predictive model. Scientific Reports, 7(1), 1-8. doi:10.1038/ s41598-017-08803-3.

Zhou, D., Lebel, C., Treit, S., Evans, A., \& Beaulieu, C. (2015). Accelerated longitudinal cortical thinning in adolescence. NeuroImage, 104, 138-145. doi:10.1016/ j.neuroimage.2014.10.005. 